Diánoia, vol. 29, no. 29, 1983

\title{
MARSILIO FICINO, O LA HUMANIZACIÓN DE LO DIVINO
}

\author{
Parte I
}

Elia Nathan

INSTITUTO DE INVESTIGACIONES FILOSǴficAs Universidad Nacional Autónoma de México

\section{INTRODUCCION}

Marsilio Ficino (1433-1494) fue el fundador de la Academia Platónica de Florencia; su gran contribución histórica radica en haber traducido al latín las obras de Platón, de los neoplatónicos, y los textos herméticos, y el haber reintroducido al pensamiento occidental la tradición platónica ([7], p. 155). Ficino también escribió tratados originales, siendo el principal su Teologia platónica sobre la inmortalidad del alma. El objetivo fundamental de ésta, al igual que el de sus otras obras, es el de armonizar lo que él consideraba las dos grandes tradiciones del pensamiento occidental, a saber, la verdadera filosofía -el platonismo- y la verdadera religión —el cristianismo ([10], pp. 90-1). Si bien durante la Alta Edad Media hubo intentos de armonización en este sentido, por ejemplo, por parte de San Agustín, la empresa ficiniana difiere de éstos tanto porque Ficino se enfrenta a la tradición escolástica-aristotélica de la Baja Edad Media, como porque entiende de otra manera a la tradición platónica, como determinada fundamentalmente por el neoplatonismo y el hermetismo.

En este trabajo intentaré presentar una línea interpretativa de las tesis fundamentales ficinianas que difiere en aspectos importantes de las interpretaciones que algunos de los principales estudiosos de Ficino han sostenido. Kristeller considera que los temas centrales de Ficino son los tres siguientes (aunque él añade un cuarto -el tema del amor platónico- que aquí no tocaré). En primer lugar, sostiene, correctamente, que el núcleo o tesis central de la filosofía de Ficino es su afirmación de que la meta última de la vida humana es el conocimiento o visión inmediata de Dios, i.e., la vida contemplativa ([2d], pp. 198 y 199). En segundo lugar, Kristeller sostiene que esta tesis de la inmortalidad está íntimamente relacionada con la tesis anterior de la vida contemplativa, por la siguiente razón: si el fin último del hombre es el conocimiento de Dios, entonces este fin debe ser conseguible no meramente por unas 
pocas personas y por cortos momentos, como sucede en la vida terrena, sino por la gran mayoría y como un estado permanente, lo cual por ser sólo posible para el alma desencarnada, presupone la inmortalidad del alma ([2d], p. 198). En tercer lugar, Kristeller sostiene que Ficino retoma de sus predecesores humanistas la doctrina de la dignidad del hombre, pero que para Ficino la dignidad del hombre radica en el hecho de que él es el centro y el cemento de la realidad, porque "para Ficino el pensamiento tiene una influencia activa sobre sus objetos (?l) y el amor, según el Banquete de Platón, es una fuerza activa que une a todas las cosas, y el alma humana extiende su pensamiento y amor a todas las cosas" ([2d], p. 197), logrando así unir a todas las cosas, i. e., ser el cemento de la realidad.

Por otra parte, Garín concuerda con Kristeller en que para Ficino el fin del hombre es el conocimiento de Dios, pero especifica un poco más en qué consiste la vida contemplativa: "La juventud florentina, habituada a que desde cátedras y discursos oficiales la exhortaran hacia la dignidad de la acción mundana y la vida civil, vio cómo cambiaba la situación y los ficinianos comenzaban a predicar las virtudes de las ascesis contemplativa." ([7], p. 139; cf., p. 146), o sea, según Garín, la vida contemplativa de la que habla Ficino ha de entenderse como desligamiento del hombre de la realidad material, como renuncia a la acción mundana (i.e., a cualquier acción terrena que no sea estrictamente necesaria para la supervivencia física o para la continuación de la vida religiosa). Esta conceptuación de la vida contemplativa la refuerza Garín sosteniendo que lo que Ficino toma de la tradición platónica es su actitud ultramundana, su énfasis en que lo verdaderamente real e importante está más allá de lo terreno o material: "La tradición platónica que le ofrecía [a Ficino] una respuesta a su pregunta más acuciante al invitarle a rebasar las apariencias de las cosas, le enseñaba al mismo tiempo a ver en la realidad circundante un símbolo alusivo que remite a otra más profunda. Aristóteles físico se detiene en la corporeidad del dato, como cualquier otro científico por lo demás." ([7], p. 149; cf., pp. 144145). Finalmente, con respecto al tema de la dignidad, que había quedado obscuro en Kristeller, Garín aclara la relación entre este tema y la vida contemplativa: la dignidad del hombre radica en su ser el cemento de la realidad, en tanto que es el pensamiento sobre, y el amor a, las cosas como manifestaciones de Dios, el que las unifica ([7], pp. 151 y 153).

Los puntos en que la interpretación de Ficino - que aquí presentarédifiere de las que acabamos de resumir, son los siguientes. En primer lugar, con respecto al problema de qué es lo que Fiçino retoma de la tradición platónica, considero que Kristeller tiene la razón, Ficino toma 
ante todo la tesis de la inmortalidad del alma - no en balde intitula a su principal obra Teología platónica sobre la inmortalidad del alma. Sin embargo, me parece que en dicha obra aparecen una serie de afirmaciones que nos permiten interpretar la tesis de la inmortalidad, no como lo hace Kristeller, a la manera tradicional, como significando que después de la muerte del cuerpo, sobrevive el alma, sino como significando algo mucho más interesante, a saber, la divinidad del alma humana. Uno de los objetivos centrales de este trabajo será precisamente mostrar qué entiende Ficino por inmortalidad o divinidad, y qué significa exactamente esto.

En segundo lugar, no concuerdo con la interpretación de Garín en el sentido de que lo que Ficino retoma del platonismo sea su actitud ultramundana ni, consecuentemente, me parece correcta su comprensión de la vida contemplativa ficiniana como una vida de desligamiento o rechazo a la vida activa mundana. Por el contrario, me parece que uno de los grandes méritos de Ficino es precisamente el haber visto que la vida contemplativa lleva a la vida activa, aun a la vida activa mundana (i.e., a la actividd en los órdenes productivo, o político, o cultural). Las razones de fondo por las que me parece incorrecta la interpretación de Garín, y correcta la interpretación contraria que he sugerido, son las siguientes. Primero, como nos recuerda el mismo Garín, para los humanistas que precedieron a Ficino, la excelencia o dignidad del hombre consistía en su actividad en el mundo ([7], p. 146), en su capacidad de transformar lo existente, creando nuevos órdenes económicos, sociales, políticos y culturales. Me parece que esta concepción del hombre como ser transformador, creativo, no podemos entenderla como una mera idea que se puso de moda durante la primera mitad del siglo $\mathrm{xv}$, sino como la concepción fundamental renacentista del hombre. $Y$ me parece que esto es así porque esta concepción del hombre surgió en el Renacimiento no como resultado de la mera dinámica de la discusión intelectual, sino como expresión intelectual de la realidad concreta que vivían los renacentistas. En efecto, la Italia del siglo $\mathrm{xv}$ es una época que se caracteriza por un gran auge económico debido a una intensa actividad comercial e industrial, por cambios sociales profundos dada la constitución de la burguesía manufacturera y comercial como una clase social con poder económico y político, por el surgimiento de las ciudades-estados en que comienza a centralizarse el poder politico, y finalmente, por un brutal desarrollo cultural (auge de las bellas artes, redescubrimiento de textos greco-latinos, invención de la imprenta, etc.). Dada esta época de gran cambio y auge a todos los niveles, resulta comprensible el que a los renacentistas el mundo les aparezca como algo digno del mayor interés, como algo valioso en sí mismo, y también el que el 
hombre les aparezca como un ser excelente en virtud de su capacidad de autodeterminarse y de determinar su entorno, creando así su propia vida; o sea, resulta comprensible por qué surgió la novedosa conciencia de la capacidad humana de re-crearse a través de su actividad mundana transformadora. ([3], p. 136). Ahora bien, dado este contexto renacentista me parece que si interpretamos a Ficino no como un pensador con una actitud ultramundana, a la manera de Garín, sino como un pensador con una actitud más mundana (actitud que se manifestaría, p.j., en su comprensión de la vida contemplativa como una vida que debe cuajar en la actividad mundana), podemos entender mejor por qué Ficino es un pensador típicamente renacentista. Cabe aclarar que lo que diferenciaría a Ficino, hombre de una profunda religiosidad, de sus predecesores, en este punto, es que Ficino no encuentra a la actividad mundana valiosa per se, como si lo hacen los primeros humanistas, sino tan sólo valiosa en la medida en que esté guiada por el conocimiento de Dios, y que por tanto sea una actividad mundana que perfecciona al hombre y al mundo. Segundo, considero que el interpretar a Ficino como un pensador que busca integrar la vida religiosa y la vida activa mundana, en contraposición a Garín, sí nos permite comprender por qué Ficino escribió un libro mágico-astrológico-religioso, De vita Triplici. En efecto, si la actividad transformadora del mundo es algo valioso para un religioso, y dado que para Ficino (al igual que para muchos pensadores renacentistas), la magia y astrología eran teorías que ofrecían el conocimiento del mundo, y por ende, podían servir para dirigir la actividad transformadora, resulta claro por qué Ficino pudo integrar la magia y astrología a un pensamiento religioso. Tercero, considero que si interpretamos a Ficino como un pensador que intenta integrar la vida contemplativa y la activa, Ficino nos aparecerá como un pensador que tiene algo importante que decirnos hoy día en la medida en que nos habla acerca del sentido que tiene para el hombre la vida activa mundana. Así, otro de los objetivos fundamentales de este trabajo será el de justificar que el intento ficiniano consiste en una armonización de la vida contemplativa y la activa a través del examen de algunos textos, y sobre todo, mediante el examen de todo un aparato metafísico que Ficino desarrolla para fundamentar dicha armonización.

Finalmente, tampoco me parece adecuada la interpretación de Garín y Kristeller de la concepción ficiniana de la dignidad del hombre en términos de su ser el nudo o punto de unión de la realidad en tanto que piensa y ama a las cosas, ya que no se entiende qué significa que el pensamiento o el mero amor sean activos por sí mismos, como dice Kristeller, o sea que transformen a la realidad. Probablemente, por el contexto en que aparece esta afirmación de Kristeller ([2d], p. 198), él pien- 
sa que dado el aprecio de Ficino a la magia y astrología, lo que Ficino quiere decir es que como hay una relación mágica entre el pensamiento y el mundo, entonces el mero pensar, o amar, afecta a los objetos materiales. Empero, me parece que esta interpretación es inadecuada por ahistórica: el que hoy día se consideren a la magia y astrología como creencias falsas o ficticias, no quiere decir que así hayan sido consideradas en el pasado; por el contrario éstas eran consideradas como conocimientos que permitían controlar el mundo de manera efectiva. Es por ello que si bien Ficino sostiene que el hombre es el punto de unión, lo es no por su pensar y amar, sino por su transformación real del mundo, basada en los supuestos conocimientos que ofrecen la magia y astrología, y guiada por el conocimiento de Dios. Es por esto último por lo que la acción transformadora del hombre tiene por fin unir "el reino de la tierra al del cielo" (i.e., tiene un fin perfeccionador).

Así pues, la línea interpretativa del pensamiento ficiniano que desarrollaré en este trabajo puede resumirse en los siguientes puntos:

1) El fin del hombre es la vida contemplativa, i.e., la unión mistica con Dios (tesis platónica).

2) El alma humana es inmortal en el sentido de que a través de dicha unión el alma se deifica o diviniza (tesis platónica).

3) La dignidad del hombre radica en la inmortalidad o divinidad de su alma, la cual debe manifestarse en la creatividad que el hombre despliega en el plano material, y en su tener una vida espiritual entendida no como la sujeción a una serie de conductas y ritos establecidos por la autoridad religiosa, y que pretenden garantizar la beatitud ultraterrena, sino como una experiencia interior que perfecciona al hombre ya desde su vida terrena.

4) Los puntos 1) y sobre todo 2) están fundados en Ficino en una concepción un tanto panteísta de Dios, de origen platónico.

Este último punto me parece muy revelador del proyecto ficiniano, ya que lo que hace la concepción panteísta de Dios es afirmar la inmanencia de Dios en el mundo, o sea, es mundanizar, y más especificamente en Ficino, humanizar lo divino. Y la razón por la cual me parece que Ficino intenta mundanizar lo divino, encontrar a Dios en la tierra, valorando así la realidad terrena, es porque su proyecto de fondo es compatibilizar una concepción religiosa de la vida humana con el espíritu laico o mundano típico del Renacimiento ([13], p. 136), espíritu que en Ficino se manifiesta en su valoración de la vida activa mundana.

Es imperativo notar que Ficino es ante todo un pensador cristiano que pretende ser ortodoxo, de tal forma que la línea interpretativa "plató- 
nica" que propongo no aparece tan nítidamente en Ficino, como notaremos a través del trabajo. A pesar de que no es la única veta del pensamiento ficiniano, me parece importante destacarla, no sólo por las razones mencionadas en la discusión previa, sino también porque considero que de esta manera puede verse a Ficino como parte de una corriente un tanto subterránea - que brota en pensadores como Cusa, Eckhart, Pico de la Mirandola, Bruno y Spinoza- de pensamiento místico de tintes panteístas, que por su búsqueda de una renovación espiritual y material del hombre, termina muchas veces por plantear cuestiones heterodoxas (tanto por ser heréticas en el plano teórico, como por la implicación anti-institucional que pueden llegar a tener).

Este trabajo se divide en dos artículos. En este primer artículo, aparecen la introducción, y un breve examen del platonismo y de la concepción ficiniana de Dios. En un artículo posterior se examinará la concepción ficiniana de la inmortalidad del alma, y de la dignidad del hombre.

\section{PLATONISMO}

Si bien, como aparece implícitamente en la Introducción, el platonismo es una corriente de pensamiento que admite interpretaciones bastante diversas, algunas de sus ideas centrales son las siguientes - nótese que estas ideas, más que constituir tesis especificas, representan una manera de comprender o aproximarse a la realidad. En primer lugar, en la metafísica de corte platónico se afirma no sólo la existencia de una realidad espiritual trascendente, sino también el que ésta determina al universo material. En segundo lugar, esta realidad trascendente tiene un carácter racional, y por ende puede ser aprehendida por el intelecto (i.e., se sostiene una posición racionalista tanto en el plano epistemológico como ontológico). En tercer lugar, se considera que la totalidad de lo existente tiene un orden jerárquico. En cuarto lugar, se afirma la inmortalidad del alma, con lo cual se quiere significar que el hombre es un viajero, más que un habitante de la tierra, ya que tiene un destino sobrenatural. Y finalmente, en quinto lugar, se piensa que el conocimiento de la Verdad (o realidad trascendente) es indispensable para guiar adecuadamente la conducta moral y la acción ([2f] pp. 334-5).

A la tradición platónica pertenece el neoplatonismo, desarrollado por pensadores como Plotino, Proclo, Iambilico, y otros, que integraron el pensamiento griego y el oriental durante los primeros siglos de nuestra era. Ahora bien, el pensamiento platónico que se desarrolló durante el Renacimiento es un pensamiento básicamente influido por el neopla- 
tonismo, el cual se caracteriza por tener un fuerte acento religioso y, más concretamente, místico ([2a], p. 177). En verdad, el neoplatonismo (sobre todo el de Plotino) consiste en una teorización a partir de la experiencia mística ([9], 18; [2g], p. 425). Hablemos, pues, un poco sobre el misticismo. El misticismo es ante todo una experiencia de, o contacto inmediato y directo con, la realidad última trascendente, la cual usualmente es percibida como la unicidad (trascendente) de todo lo existente, o sea, es la experiencia de que todas las cosas son uno, de que la multiplicidad de los seres individuales se diluye en el Uno universal ([9], pp. 18 y 46; [2c], p. 429). Dado que la experiencia mística usualmente consiste en la experiencia de la unicidad de lo existente (o, en otros términos, de la unión íntima de lo humano y lo divino), varios pensadores místicos han desarrollado una concepción panteista de Dios o Realidad trascendente - concepción consistente en considerar a Dios como la unidad de todo lo existente, o inversamente, en considerar a todo lo existente como una manifestación de Dios ([2i], pp. 32 y 35; [2c], p. 429). Hay dos vías para llegar a esta experiencia de la realidad última -nótese que ambas siempre están presentes, lo que las diferencia es que se pone más el acento en una que otra. Una vía es la del amor, que es de carácter emotiva (y a veces ritual), y la otra es la del conocimiento, que es de carácter intelectual -y podemos llamarla la vía gnóstica $([9]$, p. 40). El neoplatonismo tomó la vía gnóstica ([9], p. 18; [16], p. 12).

El misticismo se presenta en Plotino, uno de los máximos representantes del neoplatonismo, de la siguiente manera. Él considera que la realidad última y trascendente es el Uno, del cual emana el Intelecto (i.e., el mundo de las Ideas de Platón) y el Alma del Mundo (la cual crea el mundo sensible imponiéndole las Ideas a la materia informe), de tal forma que la realidad es una, pero se manifiesta en distintos grados, siendo el Superior el Uno y el inferior el mundo sensible - concepción panteísta. El Uno se manifiesta en la racionalidad, orden y armonía del mundo sensible (recuérdese que la racionalidad de Platón es, ante todo, una racionalidad matemática); y dado que estas características fueron identificadas por el pensamiento griego con el Bien, Plotino también llama al Uno el Bien ([2h], p. 280). Ahora, el fin último del hombre, para Plotino, consiste en la salvación de su alma, en su entrar a la vida eterna, y esto se logra mediante el conocimiento del Uno. Pero dado que el Uno es una unidad simple y pura, carente de toda determinación, al Uno no se le puede conocer por la razón, ya que ésta lo que conoce es lo determinado, o clasificable o conceptuable; al Uno se lo conoce por "intuición". La intuición del Uno, que es la experiencia inmediata o directa de éste, es un conocimiento tan íntimo, 
que a diferencia del conocimiento racional, el sujeto cognocente se une al objeto conocido, borrándose la diferencia entre el hombre y el Uno ([9], p. 20); o sea es la vivencia de la unidad simple y pura, carente de toda dualidad, y por ello, de la distinción hombre-Uno. Por ser la intuición del Uno el conocimiento de la unidad, en su sentido más estricto, como ausencia de toda multiplicidad y cambio, dicha intuición es difícilmente expresable en términos de la razón teórica o analítica -más adecuado le resulta el lenguaje poético; es por ello que los místicos que tratan de expresar teóricamente su conocimiento desarrollan una teología negativa. Ahora bien, si la experiencia mística es la experiencia de lo inefable, de lo innombrable, ¿por qué hemos llamado a esta experiencia 'conocimiento'? Pues bien, por una parte, porque la vivencia del Uno o Bien -esto es, la experiencia de la realidad más profunda y esencial que anima al mundo sensible - es una vivencia que transforma al hombre dándole una nueva comprensión del mundo sensible, como algo pleno de estabilidad, armonía y bondad. Y por más que esta comprensión sea inefable, es conocimiento en la medida en que guía al hombre en su relación con el mundo. Por otra parte, para llegar al conocimiento cabal del Uno se requiere de un conocimiento racional o intelectual, ya que la primera manifestación del Uno es como Intelecto, como las Ideas platónicas; y así como el Uno en orden descendente, se manifiesta como Intelecto y luego como mundo sensible, para ascender al Uno en el proceso de conocimiento, hay que ir del conocimiento sensible, al conocimiento racional, y finalmente pasar al conocimiento intuitivo, o suprarracional. Ahora bien, aunque no es muy claro cómo se pasa del conocimiento racional al intuitivo, ciertamente el paso no es deductivo o inductivo, no es racional, sino que parecería ser que consiste en un proceso, como sugiere la teología negativa, de "abstracción" ([2e], p. 511), o sea, consiste en que el conocimiento racional de las Ideas, al mostrarse a sí mismo aún como limitado en ciertos aspectos, nos incita a pasar a la aprehensión de una realidad ilimitada y absoluta. Para terminar, si bien el conocimiento filosófico (que es el que estudia a las Ideas, o más ampliamente, la racionalidad del Ser) y el conocimiento místico comparten ambos el objetivo de conocer la realidad, sin embargo, difieren no sólo por el hecho de que apelan a distintas facultades cognitivas, sino también porque al ser el conocimiento místico un conocimiento intuitivo, una experiencia o vivencia, es capaz de transformar al hombre que la ha tenido. La salvación del alma, o la participación en la vida eterna, consiste precisamente en la conciencia transformada por la experiencia mística, ya que dicha conciencia consiste en el saberse parte de un orden trascendente y superior. 


\section{LA METAFISICA DE FICINO}

En la Teologia platónica, Ficino sostuvo que hay cinco tipos de sustancias: Dios, ángeles, alma racional, cualidad y cuerpo ([3], Libro I, Cap. I, p. 79). Con respecto a esta ontología podemos decir que, aunque es cercana a la cristiana, conserva las concepciones típicamente neoplatónicas de una jerarquía de seres establecida en función de los grados de realidad que éstos tienen, grados que dependen del grado en que reflejan a las Ideas, y la concepción del alma como una entidad intermedia entre lo natural y lo divino.

Dado que Dios es el Ser Supremo para Ficino, examinaremos su concepción de Él. Notemos ante todo que el concepto ficiniano de Dios es un tanto complicado porque en él aparecen dos concepciones de Dios un tanto diversas, si no es que incompatibles, y que son la concepción neoplatónica y la cristiana.

Para la concepción neoplatónica, Dios, o sea, el Uno, es a la vez trascendente e inmanente ([11], p. 22; [14], pp. 160-1). En palabras de Ficino, "Pero este Dios es una inmensa luz, en sí y para sí, una intensísima luz que mora en todas las cosas y fuera de ellas" (Del Amor, cit., en [12], p. 154).

Dios es inmanente en tanto que es la fuente creadora de todas las cosas, y por la cual todas las cosas son una - tanto cada cosa es en sí misma una, como la totalidad de lo existente es una unidad; pero es trascendente porque la unidad del Uno, por ser simple, no se puede identificar con la unidad o colección de lo múltiple. Como dice Ficino: "Fue la obra principal del divino Platón... revelar el principio de la unidad de todas las cosas, que él apropiadamente llamó el Uno mismo. Él también afirmó que en todas las cosas hay una verdad, que es la luz del Uno mismo, la luz de Dios." ([5], Carta 42, p. 84). Pienso que esta concepción se puede aclarar si notamos que la noción de trascendencia que se utiliza aquí no significa otredad en un sentido ontológico, Dios no es una entidad distinta ontológicamente al mundo sensible; en verdad, sólo hay una única realidad, pero que se manifiesta de distintas maneras o en distintos grados, de tal forma que esta realidad, considerada como es en sí misma, es el Uno y es trascendente, pero considerada en o desde sus manifiestaciones (i.e., el mundo sensible), el Uno o la Realidad es visto como inmanente.

Esta idea de una única realidad que se manifiesta de diversas maneras, Ficino la especifica de la siguiente manera. En primer lugar, sostiene que la mente de Dios está constituida por las Ideas: "ellos aprendieron de Mercurio Trimegisto [a quien Ficino supone el autor de los textos herméticos pertenecientes a la tradición platónica], el más sabio de todos 
los egipcios, que Dios es la fuente suprema y la luz dentro de la cual brillan los modelos de todas las cosas, y a los que llaman ideas". ([5], Carta 7, p. 43). En segundo lugar, Ficino considera a los seres existentes como reflejo de dichas Ideas (y por lo cual, Dios es inmanente):

Dichas pinturas [i.e., copias de las Ideas] reciben el nombre de ejemplares e ideas en los ángeles, de razones y noticias en las almas, de imágenes y formas en la materia del mundo. Estas pinturas son claras en el mundo, más claras en el alma, y clarísimas en los ángeles. Por tanto, un mismo rostro de Dios se refleja en tres espejos dispuestos ordenadamente, en el ángel, en el alma, y en el cuerpo mundano (Del Amor, cit., en [7], pp. 150-1; cf., también [3], Libro II, cap. VII, p. 165).

Cabe aclarar aquí que hemos manejado dos versiones un poco distintas de la concepción platónica o panteísta de Dios. Por una parte, entendiendo estrictamente la concepción del Uno como la única realidad, que se manifiesta de distintas maneras, la metáfora adecuada seria, siguiendo una analogía que a veces Ficino utiliza, el considerar que Dios es como el Sol, que es la fuente de la luz, de tal forma que en él la luz se presenta con mayor intensidad, mientras que el mundo sensible es como la luz cuando está lejos del sol, que es la misma pero presente con menor intensidad. Por otra parte, al utilizar la metáfora del mundo sensible (el alma, etc.), como "espejo" de Dios, parecería ser que se introduce subrepticiamente un nuevo principio constitutivo del mundo sensible, llamémosle "materia prima", y que es en lo que se refleja Dios, de tal forma que la realidad ya no sería en sentido estricto una sola, sino que estaría constituida por dos principios ontológicos, el Uno y la materia prima. Esta segunda versión también es panteísta o inmanentista, en tanto que Dios es parte del mundo sensible que lo refleja, pero es un panteísmo menos radical que el anterior.

Es importante notar que estas dos versiones del panteísmo, que es una tesis ontológica, pueden asociarse, sin que haya ninguna relación lógica, con distintas posiciones o actitudes en otros campos. En el campo de la ética, el panteísmo no-radical parecería poderse asociar con la posición maniqueista, según la cual hay dos principios positivos o reales, el del bien y el del mal, correspondientes a los principios ontológicos. del Uno o el Bien, y la materia prima o fuente de irrealidad. En cambio, el panteísmo radical parece ir asociado con la posición más optimista de que sólo existe el bien como algo positivo, mientras que el mal no es sino ausencia del bien. Igualmente, podemos asociar las dos versiones del pantésmo con actitudes generales frente al mundo sensible; el panteísta radical tendría una actitud más optimista o positiva frente af 
mundo sensible, una actitud de apertura e interacción con éste, ya que lo vería como una manifestación divina, mientras que un panteísta no-radical podría (aunque no necesariamente, como lo muestra el caso de Plotino) tener una actitud de rechazo al mundo sensible, por considerar que éste no es Dios, o porque éste contiene un principio de irrealidad (o de maldad). Estas dos actitudes frente al mundo sensible también son actitudes frente a Dios: el optimista busca conocer a Dios por interés en, o amor a la vida terrena, porque quiere conocer la verdad más profunda de la vida, mientras que el pesimista (que hemos asociado con el panteísta no-radical) busca a Dios para huir (tanto intelectual como prácticamente) del mundo.

Ficino no hace todas estas distinciones, ni muchas otras, ya que es un pensador básicamente sincrético, y lo cual hace que su pensamiento no sea por completo coherente, sino que esté lleno de tensiones. Considerando en particular la distinción entre la actitud optimista y la pesimista, podemos decir lo siguiente. La introducción del pensamiento panteísta durante el Renacimiento tuvo por efecto justificar una actitud más optimista, o positiva frente al mundo sensible, que la medieval, dado que permitía desplazar el interés humano, antes centrado en lo ultraterreno, hacia el hombre y la naturaleza ([11], p. 46) por considerarse a éstos en algún sentido divinos, o sea, porque mundanizaba lo divino. Ahora bien, Ficino desarrolló una teoría un tanto panteísta, precisamente porque el panteísmo le permitía afirmar de una manera categórica el valor intrínseco del hombre y la naturaleza acorde con el espíritu de su época. Ficino también desarrolló una teoría con acentos panteistas porque ésta le parecía, al igual que a otros pensadores, ser una concepción que expresa mejor la experiencia mistica de Dios ([2i], p. 32). En verdad, Ficino tomó de la tradición neoplatónica no sólo tesis específicas sino el interés que está tras de ellas, y que es afirmar la relación mística con Dios, ya que él intentaba abogar (frente a la Iglesia decadente del siglo $\mathrm{xv}$ ) por una religión entendida más como una experiencia interior que perfecciona al hombre, que como formas exteriores - -esta comprensión de la religiosidad como experiencia mística, me parece, también cumple el objetivo de realzar el valor intrínseco del hombre individual, en la medida en que la religiosidad no se concibe como dependencia del hombre en una revelación y autoridad institucional que le determinan sus normas de vida, sino como vivencia personal. Empero, Ficino no llegó a ser un panteísta radical porque esta posición es considerada como herética por la Iglesia católica, y Ficino, al igual que la mayoría de los renacentistas del siglo $\mathrm{xv}$, pretendian afirmar la dignidad del hombre y la naturaleza como cristianos ortodoxos. Por otra parte, en Ficino a veces encontramos una posición más pesimista 
(como veremos más adelante). Me parece que esto se debe a que, por un lado, es un renacentista, y por ende, optimista, pero que, por otro lado, como él dice, tiene un carácter saturnino, es decir, melancólico o depresivo ([6], Carta 24, p. 33), con un mirar al mundo, a veces, muy doliente. En el resto de este artículo, enfatizaré la posición optimista, ya que no sólo me parece la más interesante desde el punto de vista vivencial, sino también, desde el punto de vista intelectual, por ser la más difícil de desarrollar.

Veamos ahora brevemente la concepción cristiana de Dios. Para ésta, Dios es trascendente no sólo en el sentido de que Dios y mundo son entidades ontológicamente distintas, sino que Dios es superior al mundo, y todas las creaturas, inclusive el hombre, están subordinadas a él ([11], pp. 52 y 63). Empero, Dios guarda una íntima relación con el mundo en tanto que lo guía, lo cuida, lo ama, y lo salva, actividades todas ellas que se centran sobre todo en el hombre, ya que éste es el ser superior entre todas las creaturas. Ahora bien, el Dios cristiano es un Dios "antropomórfico" (a diferencia del platónico, que es impersonal o abstracto), ya que sus propiedades son como las humanas, aunque superiores -es omnisapiente, omnipotente, todo amoroso y misericordioso, etc. Es por ello que la unión mística con Dios es usualmente concebida por el cristianismo como una relación amorosa entre dos personas, pero sea una unión amorosa, o intuitiva (como generalmente sostienen los pensadores platónicos), lo que es esencial para la concepción cristiana es que en dicha unión el hombre no pierde su individualidad ([16], p. 90) -a diferencia de los místicos de corte platónico, y sobre todo, de los orientales, para los que la unión consiste en que el yo se deifica, se identifica con Dios o el Uno. Por otra parte, si bien Dios expresa su interés por su creación a través de cuidarla, amarla, etc., también ha expresado su interés, y de una forma categórica, encarnándose en Cristo; y dado que la Iglesia es la heredera de Cristo, la relación del hombre con Dios debe estar mediada por la Iglesia (a diferencia de la concepción platónica que es mucho más individualista).

El concepto que de Dios tiene Ficino es parcialmente cristiano, ya que concibe a Dios antropomórficamente, como aquel que guía, es providencial, ama a sus creaturas, etc. (Theol. Pl., Libro II, Caps. VII-XIII; [1], p. 35; [10], pp. 94-95). Sin embargo, también es un concepto panteísta (i.e., Ficino modifica la concepción cristiana en un sentido platónico) porque considera que Dios es el Uno ([3], Libro I, Cap. VI, p. 167, etc.), porque sostiene que Dios contiene a las Ideas en su mente, porque tiende a concebir a Dios como inmanente (como "aquel que mora en todas las cosas"), y finalmente, porque concibe al alma humana como un ser divino. 
Como conclusión a esta primera parte, podemos decir que hemos sugerido una línea interpretativa del pensamiento de Ficino en que lo que se destaca como una veta fundamental de su pensamiento es su platonismo. Y esto porque son sus tesis de origen platónico las que fundamentan en Ficino su concepción renacentista del hombre como un ser digno en tanto que autónomo, o sea, en tanto que el hombre es capaz de determinarse a sí, ya sea en el sentido de elegir (en función de su conocimiento y experiencia) el modo de vida que le parece el más perfecto, como también en el sentido de plasmar en la realidad dicha elección a través de su transformación del mundo (i.e., de su vida activa). Las tesis o concepciones de origen platónico que le sirven para fundamentar dicha concepción del hombre son: a) una concepción mística de la religiosidad, de acuerdo con la cual el conocer a Dios es una vivencia, una experiencia personal de descubrimiento de lo real y profundo, y no una serie de creencias teóricas y/o sujeción a normas de conducta determinadas por la autoridad; y es el conocimiento místico de Dios el que sirve de guía para la acción del hombre en el mundo. b) Una concepción un tanto panteísta de Dios, que al mundanizar o humanizar a lo divino, permite tanto afirmar la posibilidad del conocimiento místico (i.e., inmediato y directo) de Dios, como también permite afirmar la dignidad del hombre como algo que debe manifestarse en la vida activa, en la interacción con el mundo material (ya que lo divino no es ultraterreno, sino terreno). c) La tesis de la mortalidad o divinidad del alma humana, según la cual es a través del conocimiento o unión mística con Dios que el hombre se diviniza. d) La divinización del alma significa que el hombre se convierte en Dios, en un ser autónomo y creador, i.e., en un ser que a través de su transformación del mundo y de sí mismo, determina su vida. Estas dos últimas tesis serán examinadas en la Parte II.

\section{BIBLIOGRAFIA}

[1] Caponigri, A. Robert. Philosophy from the Renaissance to the Romantic Age. Londres: University of Notre Dame Press. 1963.

[2] Encyclopedia of Philosophy. Ed. por Paul Edwards. Nueva York: The Macmillan Co. and The Free Press. 1967.

a) Gilbert, Neal W. "Renaissance". Vol. 7.

b) Harris, H.S. "Italian Philosophy". Vol. 4.

c) Hepburn, R. W. "Mysticism, nature and assesment of". Vol. 5.

d) Kristeller, P. O. "Ficino Marsilio". Vol. 3.

e) Osborn, E. F. "Pseudo-dionysius". Vol. 6.

f) Rees, D. A. "Platonism and the Platonic Tradition", Vol. 6.

g) Smart, Ninian. "Mysticism, History of". Vol. 6. 
h) Lewis, H. D. "Philosophy of Religion, History of". Vol. 6.

i) MacIntyre, Alasdair. "Pantheism". Vol. 6.

[3] Ficino, Marsilio. Teologia Platónica. Vol. I. Bologna: Zanichelli editore. 1965.

[4] Ficin, Marsile. Theologie Platonicienne de l'Inmortalite des Âmes. Tome II. Paris: Société d'édition "Les Belles Letheres". 1964.

[5] Ficino, Marsilio. The Letters of Marsilio Ficino. Vol. I. London: Shepheard-Wolwyn. 1975.

[6] Ficino, Marsilio. The Letters of Marsilio Ficino. Vol. II. London: Shepheard-Wolwyn. 1978.

[7] Garín, Eugenio. La Revolución Cultural del Renacimiento. Barcelona: Editorial Crítica. 1981.

[8] Hale, J. R. La Europa del Renacimiento. 1480-1520. México: Siglo XXI Eds. 1980.

[9] Happold, F. C. Mysticism, A Study and an Anthology. Middlesex: Peguin Books. 1963.

[10] Kristeller, Paul Oscar. Renaissance Thought II. Papers on Humanism and the Arts. Nueva York: Harper Torchbooks. 1965.

[11] Molnar, Thomas. God and the Knowledge of Reality. Nueva York: Basic Books, Inc. 1973.

[12] Mondolfo, Rodolfo. Figuras e ideas de la filosofía del renacimiento. Barcelona: Icaria Editorial, S. A. 1980.

[13] Romano, R. y A. Tenenti. Los fundamentos del mundo moderno. México: Siglo XXI Eds. 1981.

[14] Spencer, Sydney. Mysticism in World Religion. Middlesex: Penguin Books. 1963.

[15] Trinkaus, Charles. In our Image and Likeness. Humanity and Divinity in Italian Humanist Thought. Vol. 2. Chicago: University of Chicago Press. 1970.

[16] Wallis, R. T. Neoplatonism. Londres: Duckworth. 1972.

[17] Yates, Frances A. Giordano Bruno and the Hermetic Tradition. Chicago: University of Chicago Press. 1978. 